\title{
Effect of an Adsorbed Atom on Resonant Charge Exchange in Atom Scattering on Metal Surfaces
}

\author{
R. Taranko, E. Taranko and M. Wiertel \\ Institute of Physics, M. Curie-Skłodowska University \\ pl. M. Curie-Skłodowskiej 1, 20-031 Lublin, Poland
}

(Received January 17, 2003; revised version April 16, 2003)

\begin{abstract}
A simple model describing resonant charge transfer between the free-electron metal surface and an atom colliding with it at some distance from another adsorbed atom is presented. The negative ionization probability of a scattered atom is studied within the time-dependent Anderson-Newns model and the time-evolution operator approach. With appropriate models for the considered system, the ionization probability is shown to oscillate with the distance between the adsorbed atom and the collision point of the scattered atom with the metal surface. These oscillations are results of the indirect interaction between the adsorbed and the scattered atoms due to the coupling of both atoms with the metal energy band.
\end{abstract}

PACS numbers: 79.20.-m, 82.65.+r, 34.70.+e

\section{Introduction}

Charge-exchange processes play the fundamental role in the atom-metal surface interactions. The study of the charge transfer processes that can occur when an atom (ion) collides with the solid surface is especially of a fundamental interest in the context of many surface analysis techniques [1-3]. In many cases the resonant electron transition between the moving (scattered) atom level and the metal surface levels has the highest efficiency and this kind of electron transitions determines the final electron occupancy of the outgoing atom. The strength of the electron transfer is very sensitive to the electronic characteristics of the complex system "moving atom + metal surface", particularly it depends on the relative energy position of the atomic level and the Fermi level of the surface energy band, as 
well as, on the coupling strength between the atom and metal surface (e.g. [4-6]). Therefore, the additional modification of the surface due to the adsorbed atoms (adatoms) influences very distinctly the charge transfer and can change dramatically the final charge state of the outgoing atom beam (e.g. $[7,8]$ ).

First of all, the adsorbed atoms on a metal surface result in a change of the work function, which is a very important characteristics of the metal surface. Depending on the work function the atomic level can be localized above or below the Fermi level and thus resulting in a charge transfer. It is the so-called non-local effect as in this case the change of the metal surface characteristics concerns the entire surface and not only the region around the adsorbed atom. However, the adsorbed atom modifies locally the electronic potential and influences the level energies and coupling between the moving atom and the surface (e.g. [3, 9-13]). In addition, in the case when the moving atom is backscattered from an adsorbed atom or from the surface in the immediate vicinity of the adsorbate, then there are two different channels for electron transfer between the moving atom and the surface. The first one corresponds to direct tunneling between an atom and the surface and the second one to tunneling through the adsorbed atom level. Let us note that a similar situation is observed in the case of the scanning tunneling microscopy (STM), where there are also two different electron tunneling channels between the microscope tip and the surface with the adsorbed atom [14-16] (see also [17]).

The interaction between the adsorbed and moving atoms is usually short-ranged and for the atom-surface collision region lying further away from the adsorbed atom, this interaction is negligibly small. However, it does not mean that the charge transfer between the moving atom and the surface is the same as in the scattering in the case of clean surface. Note that the adsorbed and moving atoms are coupled to the substrate and can interact via the substrate-metal electron band (during the period when the scattered atom is in the vicinity of the surface). This indirect interaction should be dominated in a long range and is expected to be of oscillatory character (like the RKKY interaction between impurities in bulk metals (e.g. [18]). The similar indirect interaction is responsible for oscillatory behaviour of the interaction energy of two adsorbed atoms (depending on the distance between them) (e.g. [19, 20]). On the other hand, in many experiments by STM with single adsorbed atoms on metal surfaces the adsorbate-induced charge density oscillations around the adatom were observed [21, 22].

In this paper we present an attempt of theoretical evaluation of the charge state of an atom scattered from the surface when the collision region is in some distance from the adsorbate atom. The indirect interactions, which play an important role in the chemisorption or in mesoscopic physics can be, in principle, visible also in dynamical charge exchange processes at the solid surface, although the period of time during which the scattered atom is near the surface is relatively short. Particularly in the case when the adatom-metal interaction is relatively large, the 
indirect interaction between moving and adsorbed atoms can affect the charge transfer even if the direct tunneling through the adatom level is negligible. In literature there are only a few theoretical treatments of similar problems [3, 9, 10]. Borisov and Gauyacq [10] considered the neutralisation of $\mathrm{Li}^{-}$ions backscattered from the $\mathrm{Al}(100)$ surface depending on the distance of the collision region from the adsorbed $\mathrm{Cs}$ atom. Using the multi-channel rate equation approach and taking into consideration the changes of the local electrostatic potential due to the adsorbed atom and its influence on the energy shift and broadening of atomic levels the authors showed the importance of the local effect. The perturbation introduced by the adsorbed Cs atom was seen to extend to a very large distance. In their treatment the resonant charge transfer was considered for one specific system. In our paper we are going to consider the related problem using the model Hamiltonian and put an emphasis on the charge transfer calculated for a broad class of parameters characterizing the system under consideration, e.g. the atom velocity, the width of the surface electron band, the relative position of the Fermi energy level and adatom and moving atom energy levels, the values of the coupling between all parts of the system. The starting point to describe the resonant charge transfer will be the time-dependent Anderson-Newns (AN) Hamiltonian. Several authors have reviewed the resonant charge transfer processes in the framework of this model (e.g. [1, 4]). Usually, the evolution of the scattered atom is well described by a classical trajectory given by a time function $z(t), z$ being the distance between an atom and the metal surface, so the $z$-dependent parameters of the AN Hamiltonian can be easily converted into the time-dependent ones. In our calculations we renounced a wide-band limit approximation [1, 4] often used for simplified description of the metal surface electron band structure and assumed the free-electron band structure. It will be shown that for some values of parameters the oscillatory dependence of the ionization probability of the scattered atom against the distance of the collision region from the adsorbed atom is observed - the effect of the indirect interaction between the adsorbed and the scattered atoms.

The article is structured as follows: in Sec. 2 we will discuss the theoretical background while in Sec. 3 we will show the numerical results obtained and present our conclusions.

\section{Model and approximation}

We describe the system of the moving atom and a clean metallic surface with a single atom (adatom) adsorbed on it at the point $\boldsymbol{R}_{\mathrm{a}}$ by the time-dependent Anderson-Newns (AN) Hamiltonian [1, 4] (c.f. periodic AN model [23]):

$$
\begin{aligned}
H= & \sum_{\boldsymbol{k}} \varepsilon_{\boldsymbol{k}} n_{\boldsymbol{k}}+\varepsilon_{0}(t) n_{0}+\varepsilon_{\mathrm{a}} n_{\mathrm{a}}+\sum_{\boldsymbol{k}} \mathrm{V}_{\mathrm{a} k} \exp \left(\mathrm{i} \boldsymbol{k} \cdot \boldsymbol{R}_{\mathrm{a}}\right) C_{\mathrm{a}}^{+} C_{k}+\text { h.c. } \\
& +V_{0 \mathrm{a}}(t) C_{0}^{+} C_{\mathrm{a}}+\text { h.c. }+\sum_{\boldsymbol{k}} V_{0 \boldsymbol{k}}(t) C_{0}^{+} C_{\boldsymbol{k}}+\text { h.c. }
\end{aligned}
$$


where for simplicity, the spin variables are neglected and $C_{\mathrm{a}}, C_{0}, C_{\boldsymbol{k}}\left(C_{\mathrm{a}}^{+}, C_{0}^{+}, C_{\boldsymbol{k}}^{+}\right)$ are the annihilation (creation) operators for the adsorbed atom orbital, the incident atom orbital, and the surface electron state orbital, respectively. We place the origin of the coordinate system at a point on which the moving atom is scattered on the surface and we assume that the atom moves along the classical trajectory perpendicular to the surface.

The functions $V_{0 \mathrm{a}}(t), V_{\mathrm{a} \boldsymbol{k}}$, and $V_{0 k}(t)$ are the matrix elements of interactions between the moving and adsorbed atoms, and between the adsorbed and moving atoms and $\boldsymbol{k}$-th level of the surface electron band, respectively. The incident atom (labelled by a number "0") and the adatom (labelled by a letter "a") are described by its electron levels $\varepsilon_{0}(t)$ and $\varepsilon_{\mathrm{a}}$ and the surface electron band is described by $\varepsilon_{\boldsymbol{k}}$. The corresponding electron wave functions are denoted as $|0\rangle,|\mathrm{a}\rangle$, and $|k\rangle$, respectively.

We calculate the occupancy of the moving atom in terms of the time-evolution operator $U\left(t, t_{0}\right)$ (in the interaction representation) given by the equation of motion (e.g. $[25,26])$

$$
\mathrm{i} \frac{\partial U\left(t, t_{0}\right)}{\partial t}=\tilde{V}(t) U\left(t, t_{0}\right)
$$

where

$$
\tilde{V}(t)=U_{0}\left(t, t_{0}\right) V(t) U_{0}^{+}\left(t, t_{0}\right)
$$

and

$$
U_{0}\left(t, t_{0}\right)=\exp \left(\mathrm{i} H_{0}\left(t-t_{0}\right)\right)
$$

Here $H_{0}$ denotes the three first terms of Eq. (1) and $V(t)$ corresponds to all the other terms of the Hamiltonian (1). Once the appropriate matrix elements of the time-evolution operator $U_{i j}\left(t, t_{0}\right) \equiv\left\langle i\left|U\left(t, t_{0}\right)\right| j\right\rangle$ are found, where $|i\rangle$ and $|j\rangle$ belong to the set of basis functions $|a\rangle,|0\rangle,|k\rangle$, then the occupancy of the moving and adsorbed atoms can be given as follows (c.f. [26]):

$$
\begin{aligned}
& n_{0}(t)=n_{0}\left(t_{0}\right)\left|U_{00}\left(t, t_{0}\right)\right|^{2}+n_{\mathrm{a}}\left(t_{0}\right)\left|U_{0 \mathrm{a}}\left(t, t_{0}\right)\right|^{2}+\sum_{\boldsymbol{k}} n_{k}\left(t_{0}\right)\left|U_{0 k}\left(t, t_{0}\right)\right|^{2}, \\
& n_{\mathrm{a}}(t)=n_{0}\left(t_{0}\right)\left|U_{\mathrm{a} 0}\left(t, t_{0}\right)\right|^{2}+n_{\mathrm{a}}\left(t_{0}\right)\left|U_{\mathrm{aa}}\left(t, t_{0}\right)\right|^{2}+\sum_{\boldsymbol{k}} n_{k}\left(t_{0}\right)\left|U_{\mathrm{a} k}\left(t, t_{0}\right)\right|^{2} .
\end{aligned}
$$

We note that as we consider only one atom orbital, then $n_{0}(t)$ corresponds to the probability of the negative ionization (the occupancy of the affinity level) if $\varepsilon_{0}$ denotes the energy of the affinity level. In the case, where $\varepsilon_{0}$ corresponds to the atom ionization level, the probability of the positive ionization is given by $1-n_{0}(t)[26]$.

It follows from Eqs. $(5,6)$ that the occupancy of the adatom and the moving atom can be simply calculated if the appropriate matrix elements of the evolution operator are found. After some algebra it can be shown that these elements satisfy the corresponding set of coupled integro-differential equations (c.f. [25]): 


$$
\begin{aligned}
& \frac{\partial}{\partial t} U_{00}\left(t, t_{0}\right)=-\mathrm{i} \tilde{V}_{0 \mathrm{a}}(t) U_{\mathrm{a} 0}\left(t, t_{0}\right) \\
& \quad-\int_{t_{0}}^{t} \mathrm{~d} t^{\prime}\left[\Phi_{1}\left(t, t^{\prime}\right) U_{00}\left(t^{\prime}, t_{0}\right)+\Phi_{2}\left(t, t^{\prime}\right) U_{\mathrm{a} 0}\left(t^{\prime}, t_{0}\right)\right], \\
& \frac{\partial}{\partial t} U_{\mathrm{a} 0}\left(t, t_{0}\right)=-\mathrm{i} \tilde{V}_{0 \mathrm{a}}^{\star}(t) U_{00}\left(t, t_{0}\right) \\
& \quad-\int_{t_{0}}^{t} \mathrm{~d} t^{\prime}\left[\Phi_{3}\left(t, t^{\prime}\right) U_{00}\left(t^{\prime}, t_{0}\right)+\Phi_{4}\left(t, t^{\prime}\right) U_{\mathrm{a} 0}\left(t^{\prime}, t_{0}\right)\right]
\end{aligned}
$$

with the initial conditions $U_{00}\left(t_{0}, t_{0}\right)=1, U_{\mathrm{a} 0}\left(t_{0}, t_{0}\right)=0$.

Similar equations were also found for $U_{0 \mathrm{a}}\left(t, t_{0}\right)$ and $U_{\mathrm{aa}}\left(t, t_{0}\right)$ with the initial conditions $U_{0 \mathrm{a}}\left(t_{0}, t_{0}\right)=0, U_{\mathrm{aa}}\left(t_{0}, t_{0}\right)=1$, and the corresponding set of equations for $U_{0 \boldsymbol{k}}\left(t, t_{0}\right)$ and $U_{\mathrm{a} \boldsymbol{k}}\left(t, t_{0}\right)$ is as follows:

$$
\begin{aligned}
& \frac{\partial}{\partial t} U_{0 \boldsymbol{k}}\left(t, t_{0}\right)=-\mathrm{i} \tilde{V}_{0 \boldsymbol{k}}(t)-\mathrm{i} \tilde{V}_{0 \mathrm{a}}(t) U_{\mathrm{a} \boldsymbol{k}}\left(t, t_{0}\right) \\
& \quad-\int_{t_{0}}^{t} \mathrm{~d} t^{\prime}\left[\Phi_{1}\left(t, t^{\prime}\right) U_{0 \boldsymbol{k}}\left(t^{\prime}, t_{0}\right)+\Phi_{2}\left(t, t^{\prime}\right) U_{\mathrm{a} \boldsymbol{k}}\left(t^{\prime}, t_{0}\right)\right] \\
& \frac{\partial}{\partial t} U_{\mathrm{a} \boldsymbol{k}}\left(t, t_{0}\right)=-\mathrm{i} \tilde{V}_{\mathrm{a} \boldsymbol{k}}(t)-\mathrm{i} \tilde{V}_{0 \mathrm{a}}^{\star}(t) U_{0 \boldsymbol{k}}(t) \\
& -\int_{t_{0}}^{t} \mathrm{~d} t^{\prime}\left[\Phi_{3}\left(t, t^{\prime}\right) U_{0 \boldsymbol{k}}\left(t^{\prime}, t_{0}\right)+\Phi_{4}\left(t, t^{\prime}\right) U_{\mathrm{a} \boldsymbol{k}}\left(t^{\prime}, t_{0}\right)\right]
\end{aligned}
$$

with the initial conditions $U_{\mathrm{a} \boldsymbol{k}}\left(t_{0}, t_{0}\right)=0, U_{0 \boldsymbol{k}}\left(t_{0}, t_{0}\right)=0$.

Here, the functions $\Phi_{i}\left(t, t^{\prime}\right), i=1,2,3,4$, are defined as follows:

$$
\begin{aligned}
& \Phi_{1}\left(t, t^{\prime}\right)=\sum_{k} \tilde{V}_{0 \boldsymbol{k}}(t) \tilde{V}_{0 k}^{\star}\left(t^{\prime}\right), \\
& \Phi_{2}\left(t, t^{\prime}\right)=\sum_{k} \tilde{V}_{\mathrm{a} \boldsymbol{k}}(t) \tilde{V}_{\mathrm{a} k}^{\star}\left(t^{\prime}\right) \exp \left(-\mathrm{i} \boldsymbol{k} \cdot \boldsymbol{R}_{\mathrm{a}}\right), \\
& \Phi_{3}\left(t, t^{\prime}\right)=\sum_{k} \tilde{V}_{\mathrm{a} \boldsymbol{k}}(t) \tilde{V}_{0 k}^{\star}\left(t^{\prime}\right) \exp \left(\mathrm{i} \boldsymbol{k} \cdot \boldsymbol{R}_{\mathrm{a}}\right), \\
& \Phi_{4}\left(t, t^{\prime}\right)=\sum_{k} \tilde{V}_{\mathrm{a} \boldsymbol{k}}(t)^{\star} \tilde{V}_{0 k}^{\star}\left(t^{\prime}\right),
\end{aligned}
$$

where, according to Eq. (3) one obtains:

$$
\tilde{V}_{i j}(t)=V_{i j}(t) \exp \left(\mathrm{i} \int_{t_{0}}^{t}\left(\varepsilon_{i}\left(t^{\prime}\right)-\varepsilon_{j}\left(t^{\prime}\right)\right) \mathrm{d} t^{\prime}\right)
$$

and $\varepsilon_{i}$ corresponds to $|i\rangle$.

We assume the functions $V_{0 k}(t)$ and $V_{0 \mathrm{a}}(t)$ in the form $V_{0} u_{0}(t)$ and $V_{0 \mathrm{a}} u_{0 \mathrm{a}}(t)$, respectively, where the functions $u_{0}(t)$ and $u_{0 \mathrm{a}}(t)$ describe the time-dependence of the corresponding interactions. $\left.t_{0}=0\right)$ :

The functions $\Phi_{i}\left(t, t^{\prime}\right)$ can be written as follows (we assume $V_{\mathrm{a} \boldsymbol{k}}=V_{\mathrm{a}}$ and 


$$
\begin{gathered}
\Phi_{1}\left(t, t^{\prime}\right)=\left|V_{0}\right|^{2} u_{0}(t) u_{0}\left(t^{\prime}\right) \exp \left(\mathrm{i} \int_{t^{\prime}}^{t} \varepsilon_{0}\left(t^{\prime \prime}\right) \mathrm{d} t^{\prime \prime}\right) \\
\times \int_{-\infty}^{\infty} \mathrm{d} \omega \exp (-\mathrm{i} \omega t) \sum_{\boldsymbol{k}} \delta\left(\omega-\varepsilon_{\boldsymbol{k}}\right) \\
\Phi_{2}\left(t, t^{\prime}\right)=V_{0} V_{a} u_{0}(t) \exp \left(\mathrm{i} \int_{t^{\prime}}^{t} \varepsilon_{0}\left(t^{\prime \prime}\right) \mathrm{d} t^{\prime \prime}-\mathrm{i} t^{\prime} \varepsilon_{\mathrm{a}}\right) \\
\times \int_{-\infty}^{\infty} \mathrm{d} \omega \exp (-\mathrm{i} \omega t) \sum_{\boldsymbol{k}} \delta\left(\omega-\varepsilon_{\boldsymbol{k}}\right) \exp \left(-\mathrm{i} \boldsymbol{k} \cdot \boldsymbol{R}_{\mathrm{a}}\right)
\end{gathered}
$$

and similar expressions for $\Phi_{4}\left(t, t^{\prime}\right)$ and $\Phi_{3}\left(t, t^{\prime}\right)$, respectively.

In order to proceed further the calculations we assume the free-electron energy dispersion $\varepsilon_{\boldsymbol{k}}=\hbar^{2} k^{2} / 2 m$ for the metal surface electron energy spectrum. Therefore, the integral appearing in $\Phi_{1}\left(t, t^{\prime}\right)$, the Fourier transform of the density of states, $\mathcal{D}(t)$, can be simply calculated and the second integral in $\Phi_{2}(t, t)$ can be rewritten as

$$
\frac{\frac{3}{2}\left(\hbar^{2} / 2 m\right)^{1 / 2} W^{-3 / 2} \int_{0}^{W} \mathrm{~d} \omega \exp (-\mathrm{i} \omega t) \sin \left(\sqrt{2 m W / \hbar}\left|\boldsymbol{R}_{\mathrm{a}}\right|\right)}{\left|\boldsymbol{R}_{\mathrm{a}}\right|} \equiv \mathcal{F}(t),
$$

where $W$ is the bandwidth of the surface energy spectrum $D(\omega)$ and we assume $D(\omega)=\frac{3}{2} W^{-3 / 2} \omega^{1 / 2}$ for $0 \geq \omega \geq W$ and $D(\omega)=0$ elsewhere.

Finally, the functions $\Phi_{i}\left(t, t^{\prime}\right)$ which play the role of the integral kernels in the integro-differential equations $(7-10)$ can be written as follows:

$$
\begin{aligned}
& \Phi_{1}\left(t, t^{\prime}\right)=\left|V_{0}\right|^{2} u_{0}(t) u_{0}\left(t^{\prime}\right) \exp \left(\mathrm{i} \int_{t^{\prime}}^{t} \varepsilon_{0}\left(t^{\prime \prime}\right) \mathrm{d} t^{\prime \prime}\right) \mathcal{D}\left(t-t^{\prime}\right), \\
& \Phi_{2}\left(t, t^{\prime}\right)=V_{0} V_{\mathrm{a}} u_{0}(t) \exp \left(\mathrm{i} \int_{t^{\prime}}^{t} \varepsilon_{0}\left(t^{\prime \prime}\right) \mathrm{d} t^{\prime \prime}-\mathrm{i} t^{\prime} \varepsilon_{\mathrm{a}}\right) \mathcal{F}\left(t-t^{\prime}\right), \\
& \Phi_{3}\left(t, t^{\prime}\right)=V_{\mathrm{a}} V_{0} u_{0}\left(t^{\prime}\right) \exp \left(-\mathrm{i} \int_{0}^{t^{\prime}} \varepsilon_{0}\left(t^{\prime \prime}\right) \mathrm{d} t^{\prime \prime}+\mathrm{i} t \varepsilon_{\mathrm{a}}\right) \mathcal{F}\left(t-t^{\prime}\right), \\
& \Phi_{4}\left(t, t^{\prime}\right)=\left|V_{\mathrm{a}}\right|^{2} \exp \left(\mathrm{i} \varepsilon_{\mathrm{a}}\left(t-t^{\prime}\right)\right) \mathcal{D}\left(t-t^{\prime}\right) .
\end{aligned}
$$

It is clear that the importance of the charge transfer channel between the moving atom and the metal surface through the tightly bound adatom orbital |a should decrease with the increasing distance between adatom and the point on the surface at which the moving atom scatters. In our set of equations for the matrix elements of the evolution operator (Eqs. $(7-10)$ ), the information on the relative position of the adatom and the moving atom is contained in the function $\mathcal{F}(t)$ and $u_{0 \mathrm{a}}(t)$. For a very large $\left|\boldsymbol{R}_{\mathrm{a}}\right|$ (note that we placed the origin of the coordinate system at a point on the surface where the moving atom strikes the surface) the influence of the adatom on the direct charge transfer between the metal surface and the moving atom is almost negligible and we can simplify the set of equations $(7-10)$ 
neglecting the functions $\Phi_{2}\left(t, t^{\prime}\right)$ and $\Phi_{3}\left(t, t^{\prime}\right)$. The resulting equations for $n_{0}(t)$ will be the same as in the case of atom scattering on a clean metal surface $[24,26]$.

Note, however, that at $\left|\boldsymbol{R}_{\mathrm{a}}\right|$ for which $u_{0 \mathrm{a}}(t)$ is negligible and the direct charge transfer between the adatom and the moving atom can be ignored, the functions $\Phi_{2}\left(t, t^{\prime}\right)$ and $\Phi_{3}\left(t, t^{\prime}\right)$ cannot be neglected and there is still possibility that the adatom can affect the charge transfer between the moving atom and the metal surface.

\section{Results and discussion}

We discuss the charge transfer between the moving atom and the metal surface with the adsorbed adatom as a function of the distance between the adatom and the point of the surface at which the moving atom is scattered. We assume the trajectory of the moving atom perpendicular to the surface. The velocity of the atom is assumed to be in the range of $v=0.1-0.02 \mathrm{a} . \mathrm{u}$. and is constant on the way towards the surface, as well as, on the outgoing trajectory. We calculate the charge localized on the moving atom according to Eq. (5) and the required matrix elements $U_{i j}\left(t, t_{0}\right)$ are obtained solving Eqs. $(7-10)$ and the corresponding set of equations for $U_{0 a}\left(t, t_{0}\right)$ and $U_{\mathrm{aa}}\left(t, t_{0}\right)$ (not displayed here). In order to solve these equations, the explicit forms for the time-dependence, or equivalently, the $z$-dependence of the hybridization matrix elements $V_{0 a}(z), V_{0 k}(z)$ and the $z$-dependence of the atomic energy level are required. In the literature various forms of the $z$-dependence for $V_{0 k}(z)$ have been explored - exponential, Gaussian, nodal or others. The exponential form commonly used represents fairly well the true hopping integral and should be sufficient for our model calculations. So, we characterize $V_{0 k}(t)$ through the collision time $t_{c}$, or the interaction range $\lambda_{1}$ (the collision time can be estimated as $\lambda_{1} / v$, where $\lambda_{1}$ is the interaction range and $v$ is the velocity of the moving atom $[27,28]$ ):

$$
V_{0 \boldsymbol{k}}(t)=V_{0} \exp \left(-|t| / t_{\mathrm{c}}\right)=V_{0} \exp \left(-z / \lambda_{1}\right)
$$

For numerical calculations we adopt the value $\lambda_{1} \simeq 2.5$ a.u. estimated in [28]. We adopt also the same distance dependence for the hopping integral between the moving and adsorbed atoms with $\lambda_{2} \simeq 2.5$ a.u. However, it is known that such approximation for the hopping integral works sufficiently well far away from the surface and is overestimated at distances close to the surface. Therefore, we have repeated some of our calculations with the hopping integral damped or increased in the region $0<z<2$ (in comparison with the exponential approximation). As a result, we did not find any qualitative differences in the results obtained. As for the matrix element $V_{\mathrm{a} \boldsymbol{k}}$ (it should be time-independent) we assume the sudden switching on of the interactions between the adatom and the metal surface and take it in the form $V_{\mathrm{a} k}(t)=V_{\mathrm{a}} u(t)$, where $u(t)=0$ for $t<0$ and $u(t)=1$ for $t>0$.

It is well known that, as an atom approaches the surface, the atom ionization and affinity levels are shifted by the image potential and broadened into a 
resonance of some width (e.g. [12]). The situation is more complex for the case of atoms scattered on adatoms adsorbed on the surface. The studies of the interaction of atomic levels with an adatom adsorbed on a metal surface performed in the "on top" geometry show that the atomic levels and their widths are very clearly disturbed in comparison with the results obtained for clean surfaces (e.g. $[9,29])$. These disturbances should disappear relatively fast with the increasing distance of the collision region from the adsorbed atom. In our model calculations we assumed two forms for $\varepsilon_{0}(z)$. The first one corresponds to $\varepsilon_{0}=$ const. As the main aim of this work is to investigate the dependence of the charge transfer (between a moving atom and the system consisting of the adsorbed atom and the metal surface) on the distance of the collision region from the adsorbed adatom, then such simplified assumption about $\varepsilon_{0}$ should be justified. In the second step we used the formula for $\varepsilon_{0}(z)$ consistent with the image potential correction. As expected, this improved expression for $\varepsilon_{0}(z)$ did not change the results qualitatively. Our investigations consider the negative ionized atom approaching the surface, so the occupation number $n_{0}$ corresponds to the probability of the negative ionization of the moving atom. In all figures we present results for the asymptotic values of the occupation numbers $n_{0}$ (i.e. far away from the surface) obtained for particular parameters given below in the text.

In Fig. 1 we show the occupation numbers $n_{0}$ versus the distance of the collision region from the adsorbed atom for $\varepsilon_{\mathrm{a}}$ equal to the Fermi energy $\varepsilon_{\mathrm{F}}$ and for the three constant values of $\varepsilon_{0}, \varepsilon_{0}=\varepsilon_{\mathrm{F}}-0.5 \mathrm{eV}, \varepsilon_{0}=\varepsilon_{\mathrm{F}}$ and $\varepsilon_{0}=\varepsilon_{\mathrm{F}}+0.5 \mathrm{eV}$, curves $\mathrm{A}, \mathrm{B}$, and $\mathrm{C}$, respectively. The results for $d=0$ correspond to "on top" geometry, i.e. for the collisional ion approaching the surface along the surface normal that goes through an adsorbed atom. The broad free-electron energy band with $\varepsilon_{\mathrm{F}}=11.7 \mathrm{eV}$ was assumed to mimic the aluminium surface. The parts a, b, and $c$ correspond to the ion velocities $v=0.1,0.05$, and 0.02 (in atomic units) and $V_{0}=V_{0 \mathrm{a}}=V_{\mathrm{a}}=1 \mathrm{eV}$. Generally, the occupation numbers $n_{0}$ are smaller for small $d$ in comparison with the results for larger $d$ which are essentially equivalent to those obtained for scattering on a clean surface. In all cases the existence of the efficient additional channel for electron tunneling between the collisional atom and the metal surface $\left(V_{0 \mathrm{a}} \neq 0\right)$ results in decreasing the charge localized on the scattered atoms. However, we observe some interesting interference effects which manifest in decreasing $n_{0}$ for the collision region lying in a distance from the adsorbed atom. This effect is clearly visible for smaller velocities $v=0.05$ and 0.02 , curves $\mathrm{A}$ and $\mathrm{B}$. Generally, $n_{0}(d=0)$ are smaller in comparison with $n_{0}(d=30)$ for all velocities and localizations of $\varepsilon_{0}$ and $\varepsilon_{\mathrm{a}}$. The largest charge transfer from the moving atom to the metal surface is observed for small atom velocities for the case of $\varepsilon_{0}$ localized above the Fermi energy, curve $\mathrm{C}$ in Fig. 1c. We note also for smaller atom velocities a weak dependence of the occupation numbers calculated for $d=0$ (in comparison with the results obtained for $d=20$ ) on the atom velocities and relative localization of $\varepsilon_{0}$ and $\varepsilon_{\mathrm{a}}$. 


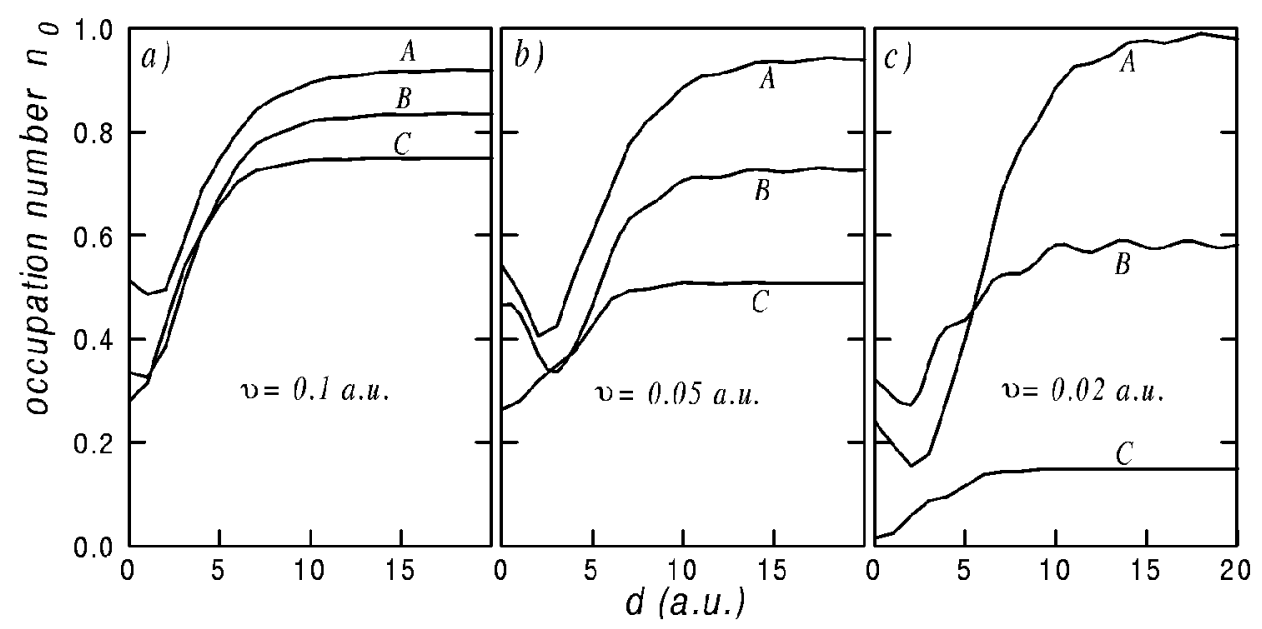

Fig. 1. Occupation numbers $n_{0}$ as the functions of a distance between the adatom and the point of the surface at which the moving atom strikes the surface, $d$, for the atom velocities $v=0.1,0.05$, and 0.02 a.u., the parts (a), (b), and (c), respectively. The curves $\mathrm{A}, \mathrm{B}$, and $\mathrm{C}$ correspond to $\varepsilon_{\mathrm{a}}-\varepsilon_{0}=0.5 \mathrm{eV}, 0 \mathrm{eV}$, and $-0.5 \mathrm{eV}$, respectively. The surface energy band is described within the free-electron model with the bandwidth $W=18 \mathrm{eV}$ and the Fermi energy $\varepsilon_{\mathrm{F}}=11.7 \mathrm{eV}$. The atomic energy level $\varepsilon_{0}$ is assumed to be independent of the distance from the surface and $\varepsilon_{\mathrm{a}}=\varepsilon_{\mathrm{F}}, V_{0}=V_{\mathrm{a}}=\mathrm{V}_{0 \mathrm{a}}=1 \mathrm{eV}$.

In Fig. 2 we show the $1 / v$ dependence of the occupation numbers $n_{0}$ calculated for different distances $d$ between a collision region and an adsorbed atom. It is well known that the resonant charge transfer between two atoms (in the absence of the surface) leads to periodic in $1 / v$ oscillations of the appropriate occupation numbers, $v$ being the relative velocity of atoms [25, 30]. Here we have extended the investigations of the $1 / v$ dependence of the occupation numbers $n_{0}$ to the case of atoms scattered on the adsorbed atom $(d=0)$ or in some distance from it. For a relatively large distance of the collision place from the adsorbed atom $(d=10$ or $d=15$ a.u.) we do not observe any oscillations independently of the strength of the hybridization constants $V_{0}, V_{\mathrm{a}}, V_{0 \mathrm{a}}$ and relative position of the energy levels $\varepsilon_{\mathrm{F}}, \varepsilon_{\mathrm{a}}$, and $\varepsilon_{0}$. The oscillations appear for an atom - adatom scattering $(d=0)$ and disappear sufficiently fast with the increasing distance $d$. Note that, surprisingly, the greater oscillations (at $d=0$ ) are observed with the weaker coupling constants $V_{0}, V_{0 \mathrm{a}}$, and $V_{0 \mathrm{a}}$ (Fig. $2 \mathrm{~b}$ ).

As the constant values for ionization or affinity levels of the moving atom are rather a crude approximation we performed also calculations of the atom occupation numbers $n_{0}$ for the case of the affinity level shifted downward as the atom approaches the surface. In Fig. 3 we plot the $d$ dependence of the occupation numbers $n_{0}$ for two values of the hybridization constants equal to $1 \mathrm{eV}$ (thick lines) and $2 \mathrm{eV}$ (thin lines) for $v=0.1$ a.u. (Fig. 3a) and $v=0.05$ a.u. (Fig. 3b). The curves 


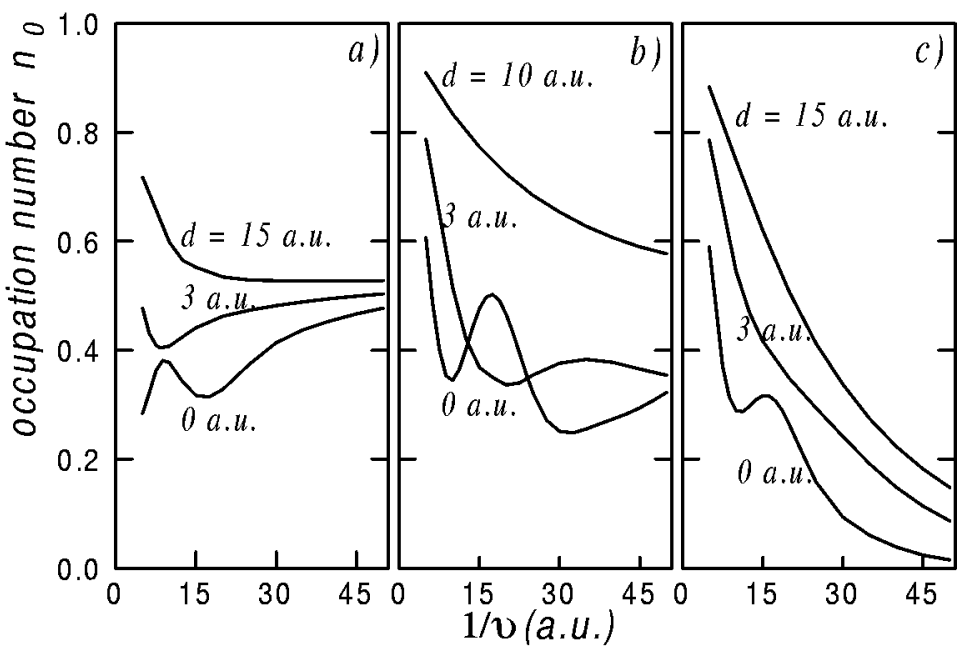

Fig. 2. Occupation numbers $n_{0}$ as the functions of $1 / v$ for $V_{0}=V_{\mathrm{a}}=\mathrm{V}_{0 \mathrm{a}}=2 \mathrm{eV}$ (part (a)) and for $V_{0}=V_{\mathrm{a}}=\mathrm{V}_{0 \mathrm{a}}=1 \mathrm{eV}$ (parts (b) and (c)). Particular curves correspond to different distances $d$. The curves denoted by $d=0$ correspond to the atom-adatom collision case. The parts (a) and (b) describe the case $\varepsilon_{0}=\varepsilon_{\mathrm{a}}=\varepsilon_{\mathrm{F}}$ and the part (c) corresponds to $\varepsilon_{0}-\varepsilon_{\mathrm{F}}=0.5 \mathrm{eV}, \varepsilon_{\mathrm{a}}=\varepsilon_{\mathrm{F}}$.

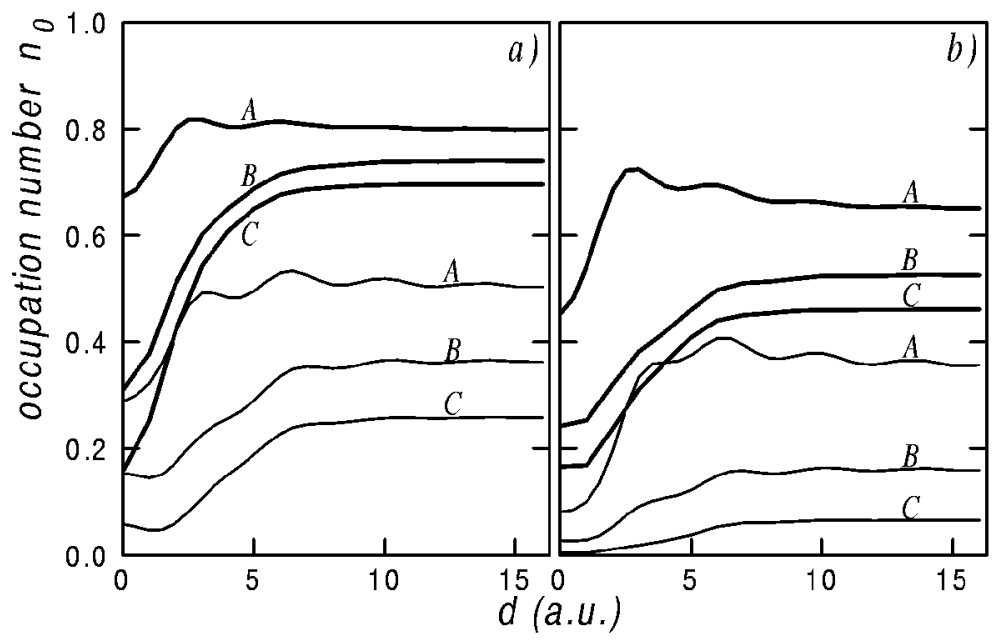

Fig. 3. Occupation numbers $n_{0}$ as the functions of a distance $d$ for the atom velocities $v=0.1$ a.u. and 0.05 a.u., parts (a) and (b), respectively. The thick (thin) lines correspond to $V_{0}=V_{\mathrm{a}}=\mathrm{V}_{0 \mathrm{a}}=1 \mathrm{eV}\left(V_{0}=V_{\mathrm{a}}=\mathrm{V}_{0 \mathrm{a}}=2 \mathrm{eV}\right)$. The atom electron affinity energy level is assumed to vary along the distance from the surface to account the image interaction. The curves $\mathrm{A}, \mathrm{B}$, and $\mathrm{C}$ correspond to the work function $\varphi=2.32 \mathrm{eV}$, $2.82 \mathrm{eV}$, and $3.5 \mathrm{eV}$, and $\varepsilon_{\mathrm{a}}=\varepsilon_{\mathrm{F}}-0.5 \mathrm{eV}, \varepsilon_{\mathrm{a}}=\varepsilon_{\mathrm{F}}$ and $\varepsilon=\varepsilon_{\mathrm{F}}+0.7 \mathrm{eV}$, respectively. 
$\mathrm{A}, \mathrm{B}$, and $\mathrm{C}$ correspond to the work function equal to $2.32 \mathrm{eV}, 2.82 \mathrm{eV}$, and $3.5 \mathrm{eV}$, respectively, and for corresponding $\varepsilon_{\mathrm{a}}$ we assumed the values $\varepsilon_{\mathrm{F}}-0.5 \mathrm{eV}, \varepsilon_{\mathrm{F}}$ and $\varepsilon_{\mathrm{F}}+0.7 \mathrm{eV}$. For this choice of parameters, the curve $\mathrm{C}$ corresponds to the affinity level $\varepsilon_{0}(z)$ lying entirely above the Fermi energy for all values of $z$. The curves $\mathrm{B}$ describe the situation when $\varepsilon_{0}(0)=\varepsilon_{\mathrm{F}}$, i.e. the affinity level is shifted downward up to the Fermi energy at $z=0$ and the curves $A$ correspond to the case for which $\varepsilon_{0}(z)$ is going down below the Fermi energy at some distance from the surface. In all cases we assumed $\varepsilon_{\mathrm{a}}=\varepsilon_{0}(0)$. These three cases A, B, C can be compared, to some extent, with the corresponding arrangement of $\varepsilon_{\mathrm{F}}, \varepsilon_{\mathrm{a}}$ and the constant values of $\varepsilon_{0}$ studied in Fig. 1. Essentially, at greater velocity the overall $d$-dependence of the occupation numbers $n_{0}$ is very similar to that given in Fig. 1. We observe greater charge transfer to the surface at small values of $d$ than for scattering on a clean surface. At smaller velocity (Fig. 3b) we still observe considerably smaller occupancies $n_{0}$ for the case of a moving atom colliding with an adsorbed atom or striking the surface at small distances from it. For stronger coupling between the moving atom, adatom and metal surface (thin curves), the enhanced charge transfer from the moving atom to the surface is observed, although for small $d$ this transfer is greater than for the clean surface.

In the next step we are going to discuss the role of the indirect interaction between the adsorbed atom and the moving atom colliding with the surface at some distance from the adsorbed atom on the charge transfer between the moving atom and the surface. This indirect interaction comes from the perturbation of the conduction electron states by the adsorbed atom. This perturbation influences the electron tunneling process between the moving atom and the surface even at the distances $d$ at which the direct electron hopping between the adsorbed and the moving atoms is negligible (c.f. $[15,16]$ ). Figure 4 presents the comparison of the occupation numbers $n_{0}$ calculated for the nonzero interactions $V_{0}, V_{\mathrm{a}}$, and $V_{0 \text { a }}$ (thick lines) and for the vanishing direct hopping $V_{0 a}$ (thin lines). We observe relatively large oscillations at small $d$ (thin curves) which are due to the indirect interaction between the moving and the adsorbed atoms. If we include the direct hopping (thick curves) then at small distances the influence of the indirect interaction is still visible although strong interference effects with direct hopping are present. For the greater distance $d$, only the oscillations due to the indirect interaction survive and the influence of the direct hopping between the adsorbed and the scattered atoms is negligible. These oscillations disappear with the increasing $d$ and finally $n_{0}$ achieves the value corresponding to scattering on clean surfaces. The influence of the indirect interaction grows up with the increasing coupling between the adsorbed atom and the surface and is greater for lower atom velocities.

Figure 5 shows the oscillations of $n_{0}$ for different values of the coupling constants $V_{0}, V_{\mathrm{a}}, V_{0 \mathrm{a}}$ for different bandwidths $W$ of the free-electron metal surface energy bands. Note the dependence of $n_{0}$ on the energy bandwidth $W$. The substrate band structure enters into the theory mainly through the time-Fourier 


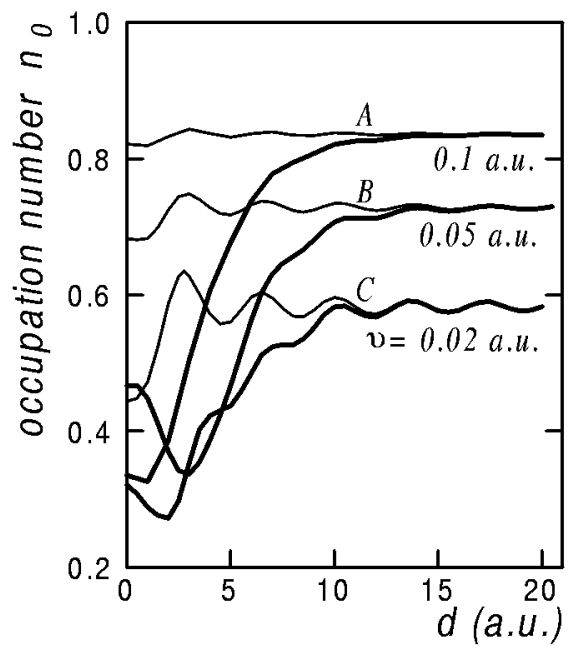

Fig. 4. Occupation numbers $n_{0}$ as the functions of a distance $d$ for different atom velocities $v=0.1$ a.u., 0.05 a.u., and 0.02 a.u. - curves A, B, and C, respectively. The thick curves correspond to $V_{0 \mathrm{a}}=V_{0}=V_{\mathrm{a}}=1 \mathrm{eV}$ and thin curves are calculated for the vanishing direct coupling between the adatom and the moving atom, $V_{0 a}=0$. Other parameters as in Fig. 1 and $\varepsilon_{0}=\varepsilon_{a}=\varepsilon_{\mathrm{F}}$.

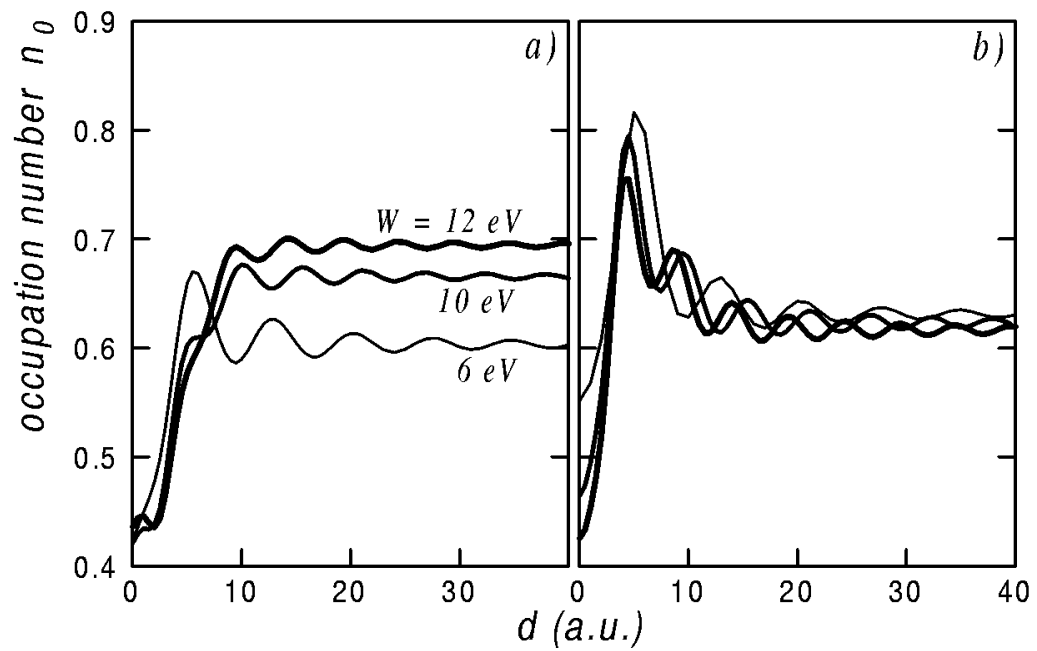

Fig. 5. Occupation numbers $n_{0}$ as the functions of $d$ for $V_{0 a}=V_{0}=V_{\mathrm{a}}=1 \mathrm{eV}$ (part (a)) and for $V_{0 \mathrm{a}}=V_{0}=V_{\mathrm{a}}=2 \mathrm{eV}$ (part (b)) for different values of the bandwidth $W$. The Fermi energy is put in the middle of the free-electron energy band and $\varepsilon_{\mathrm{a}}=\varepsilon_{0}=\varepsilon_{\mathrm{F}}$, $v=0.05$ a.u.

transform of the density of states. It seems, however, that the width of the energy band (and not the detailed structure of the energy dispersion function) is most 
important characteristic which influences the charge transfer if only the adatom's and moving atom's ionisation and affinity levels are not too close to the band edges or peaks of the density of states (e.g. [31]). Therefore in our paper we consider rather the influence of the substrate energy bandwidth than the specific energy dispersion function. For relatively small interactions between all parts of the considered system we observe a clear dependence of asymptotic values of $n_{0}$ on the width $W$ (Fig. 5a), whereas this dependence disappears for larger interactions (Fig. 5b). On the basis of the results given in Figs. 4, 5 and others we have performed, one can conclude that the period of oscillations does not depend on the atom velocity but it decreases with the increasing bandwidth $W$. The amplitude of these oscillations hardly depends on the bandwidth $W$ but increases with the decreasing atom velocity.

\section{Conclusions}

The main purpose of this work was to establish a relationship between the final charge state of the atom colliding with the metal surface in a given distance from the adsorbed atom and the indirect interaction between those atoms due to the coupling with metal electrons. Unfortunately, up to now the experimental studies only partly have addressed the problem similar to the one considered here. Namely, the experiment is able to measure the charge state of atoms backscattered from the adsorbed atoms or from the substrate atoms (e.g. [9]). However, unlike the STM where one can observe the atomic geometrical structure on surfaces and probe the surface electronic structure near the specific impurity atom, the experiments on charge exchange in the atom-surface interactions give us, e.g. the ionization probability of the scattered atom averaged over all configurations of adatoms on the surface and not for one specific surface atom placed in a definite distance from the adsorbed atom. So, our model investigations have to be considered at present time as the first step to the theoretical description of the influence of indirect interactions on charge transfer in scattering atoms on metal surfaces.

In conclusions, we have presented a study of the resonant charge transfer between a metal surface and an atom scattered on it in a distance from the adsorbed impurity atom. We have used the time-dependent Anderson-Newns model together with the evolution operator approach. The calculations of the negative ionization probability of the scattered atom were performed assuming constant, as well as, $z$-dependent (due to the image potential corrections) atom electron affinity level, for different values of the interactions between all parts of the considered system and different velocities of the moving atom. We assumed the exponential form for the hybridization matrix elements $V_{0 k}(z)$ and a free-electron band structure of the metal surface. Our main conclusions can be summarized as follows: the indirect interaction between the atoms scattered on the surface and the adsorbed impurity atoms results in oscillations of the occupation numbers $n_{0}$ of the atoms 
reflected from the surface. The occupation numbers $n_{0}$ oscillate against the distance between the adsorbed impurity atom and the collision point of a moving atom with the metal surface. These oscillations are especially clearly visible for larger couplings between atoms and the surface as well as for small atom velocities. They appear independently of the functional dependence of the atom electron energy level on the distance of this atom to the surface. The amplitude of these oscillations hardly depends on the bandwidth $W$ of the metal energy band and the period decreases with the increasing $W$.

\section{Acknowledgments}

This work has been partly supported by the State Committee for Scientific Research under Contract No. 2 P03B 01822.

\section{References}

[1] R. Brako, D.M. Newns, Rep. Progr. Phys. 53, 655 (1989).

[2] S.G. Davison, Z.L. Miskovic, A.T. Amos, B.L. Burrows, F.O. Goodman, K.W. Sulston, in: Electronic Processes at Solid Surfaces, Eds. E. Ilisca, K. Makoshi, World Scientific, Singapore 1996, p. 107.

[3] J.P. Gauyacq, A.G. Borisov, J. Phys., Condens. Matter. 10, 6585 (1998).

[4] R. Brako, D.M. Newns, Surf. Sci. 108, 253 (1981).

[5] M. Wiertel, R. Taranko, E. Taranko, Acta Phys. Pol. A 96, 769 (1999).

[6] R. Taranko, E. Taranko, Surf. Sci. 441, 167 (1999).

[7] J.J.C. Geerlings, L.F. Tz Kwakman, J. Los, Surf. Sci. 184, 309 (1991).

[8] J.N.M. van Wunnik, J.J.C. Geerlings, E.H.A. Granneman, J. Los, Surf. Sci. 131, $17(1983)$.

[9] D.G. Goryunov, A.G. Birosov, G.E. Makhmetov, D. Teillet-Billy, J.P. Gauyacq, Surf. Sci. 401, 206 (1998).

[10] A.G. Borisov, J.P. Ganyacq, Surf. Sci. 445, 430 (2000).

[11] C.B. Weare, J.A. Yarmoff, Surf. Sci. 348, 359 (1996).

[12] P. Nordlander, N.D. Lang, Phys. Rev. B 44, 13681 (1991).

[13] A.G. Borisov, G.E. Makhmetov, D. Teillet-Billy, J.P. Gauyacq, Surf. Sci. 350, L205 (1996).

[14] V. Madhavan, W. Chen, T. Jamneala, M.F. Crommie, N.S. Wingreen, Science 280, 567 (1998).

[15] A. Schiller, S. Hershfield, Phys. Rev. B 61, 9036 (2000).

[16] M. Plihal, J.W. Gadzuk, Phys. Rev. B 63, 085404 (2001).

[17] B.R. Bułka, P. Stefański, Phys. Rev. Lett. 86, 5128 (2001).

[18] R.M. White, Quantum Theory of Magnetism, Springer, Berlin 1983.

[19] T.B. Grimley, S.M. Walker, Surf. Sci. 14, 395 (1969). 
[20] T.L. Einstein, J.R. Schrieffer, Phys. Rev. B 7, 3629 (1993).

[21] M.F. Crommie, C.P. Lutz, D.M. Eigler, Science 262, 218 (1993).

[22] T. Kawasaka, H. Kasai, A. Okiji, Phys. Lett. A 250, 403 (1998).

[23] A.C. Hewson, The Kondo Problem to Heavy Fermions, Cambridge University Press, Cambridge 1993.

[24] M. Tsukada, Progr. Theor. Phys. (Suppl.) 106, 257 (1991).

[25] M. Wiertel, E. Taranko, R. Taranko, Acta Phys. Pol. A 101, 837 (2002).

[26] T.B. Grimley, V.C. Jyothi Bhasu, K.L. Sebastian, Surf. Sci. 124, 305 (1983).

[27] M. Kato, D.J. O'Connor, R.J. MacDonald, Nucl. Instrum. Methods B 78, 77 (1993).

[28] M. Kato, D.J. O'Connor, K. Yamamoto, R. Souda, Surf. Sci. 363, 150 (1996).

[29] A.G. Borisov, G.E. Makhmetov, D. Teillet-Billy, J.P. Gauyacq, Surf. Sci. 375, L367 (1997).

[30] W. Bloss, D. Hone, Surf. Sci. 72, 277 (1978).

[31] S. Tsuneyuki, N. Shima, M. Tsukada, Surf. Sci. 186, 26 (1987). 